\title{
Derivation of Schrödinger equation from a variational principle
}

\author{
Sami. H. Altoum \\ Umm Al-qura University -KSA-University College of Al- Qunfudah, Zip code 21912 - box 1109
}

Email address:

samialtoum@hotmail.com

\section{To cite this article:}

Sami. H. Altoum. Derivation of Schrödinger Equation from a Variational Principle. Pure and Applied Mathematics Journal. Vol. 2, No. 4, 2013, pp. 146-148. doi: 10.11648/j.pamj.20130204.12

\begin{abstract}
The aim of this research is to derive Schrödinger equation from calculus of variations (variational principle), so we use the methodology of calculus of variations. The variational principle one of great scientific significance as they provide a unified approach to various mathematical and physical problems and yield fundamental exploratory ideas.
\end{abstract}

Keyword: Schrödinger Equation, Variatinal Principle, Hamiltonian-Jacobi Equation

\section{Introduction}

The calculus of variations is a field of mathematical analysis that deals with maximizing or minimizing functional, which are mappings from a set of functions to the real numbers. Functional are often expressed as definite integrals involving functions and their derivatives. The interest is in extremal functions that make the functional attain a maximum or minimum value or stationary functions, those where the rate of change of the functional is zero. In quantum mechanics, the Schrödinger equation is a partial differential equation that describes how the quantum state of some physical system changes with time. It was formulated in late 1925, and published in 1926, by the Austrian physicist Schrödinger. In classical mechanics, the equation of motion is Newton's second law, and equivalent formulations are the Euler-Lagrange equations and Hamilton's equations. In all these formulations, they are used to solve for the motion of a mechanical system, and mathematically predict what the system will do at any time beyond the initial settings and configuration of the system. In quantum mechanics, the analogue of Newton's law is Schrödinger's equation for a quantum system, usually atoms, molecules, and subatomic particles; free, bound, or localized. It is not a simple algebraic equation, but (in general) a linear partial differential equation. The differential equation describes the wave function of the system, also called the quantum state or state vector. In this research we deduced Schrödinger equation from varitional principle so we introduce Schrödinger equation, canonical equations and variational problems and the Hamiltonian-Jacobi equation

\subsection{1-Canonical Equations and Variational problems}

In several problems of physics and mechanics it is convenient to recast Euler's equations in canonical form, which makes possible a general approach to variational problems. Further, the new variable introduced in the process admit of a simple physical interpretation.

Consider the extremum of the functional

$$
I\left[y_{1}, y_{2}, \ldots, y_{n}\right]=\int_{x_{1}}^{x_{2}} F\left(x . y_{1}, y_{2}, \ldots, y_{n}, y_{1}^{\prime}, y_{2}^{\prime}, \ldots, y_{n}^{\prime}\right) d x
$$

Where $y_{1}(x), \ldots, y_{n}(x)$ satisfy certain boundary conditions at $x_{1}$ and $x_{2}$.The Euler equations are

$$
F_{y_{i}}-\frac{d}{d x} F_{y_{i}^{\prime}}=0 \quad i=1.2, \ldots, n
$$

Which constitute a system of $n$ ordinary differential equation in $y_{1}(x), \ldots, y_{n}(x)$. We introduce

$$
p_{i}=F_{y_{i}^{\prime}}\left(x, y_{1}, \ldots, y_{1}^{\prime}, \ldots, y_{n}^{\prime}\right) i=1.2, \ldots, n
$$

Which together with $y_{i}(i=1.2, \ldots, n)$ are called canonical variables for the above functional. The variables $y_{i}$ and $p_{i}$ are known as canonically conjugate variables. Then (2) gives 


$$
\frac{d p_{i}}{d x}=\frac{\partial F}{\partial y_{i}} i=1.2, \ldots, n
$$

Now, if the Jacobian

$$
\frac{D\left(F_{y_{1}^{\prime}}, F_{y_{2}^{\prime}}, \ldots, F_{y_{n}^{\prime}}\right)}{D\left(y_{1}^{\prime}, y_{2}^{\prime}, \ldots, y_{n}^{\prime}\right)} \neq 0 \text {, }
$$

Then the system of equation (3) can be solved as

$$
y_{i}^{\prime}=\omega_{i}\left(x, y_{1}, \ldots, y_{n}, p_{1}, \ldots, p_{n}\right)
$$

When these are substituted into (4), we get a system of first-order equations as

$$
\frac{d y_{i}}{d x}=\omega_{i}\left(x, y_{1}, \ldots, y_{n}, p_{1}, \ldots, p_{n}\right), \frac{d p_{i}}{d x}=\frac{\partial F}{\partial y_{i}} \text { with } i=1.2, \ldots, n .
$$

Henceforward the parentheses in the second equation of (5) signify that $y_{i}^{\prime}$ in $F$ are replaced by $\omega_{i}$.We now introduce the Hamiltonian function

$$
H\left(x, y_{1}, \ldots, y_{n}, p_{1}, \ldots, p_{n}\right)=\sum_{i=1}^{n} \omega_{i} p_{i}-F
$$

Then the system (6) can be written as

$$
\frac{d y_{i}}{d x}=\frac{\partial H}{\partial p_{i}}, \frac{d p_{i}}{d x}=-\frac{\partial H}{\partial y_{i}} \quad i=1.2, \ldots, n
$$

This system is referred to as the Hamiltonian (canonical) system of Euler's equations and of 2 -ordinary equations in 2n unknown functions $y_{i}(x)$ and $p_{i}$.

\subsection{2-The Hamiltonian-Jacobi Equation}

Consider the functional in (1), the Euler equation for this functional admit of solutions involving $2 \mathrm{n}$ arbitrary constants. Here specification of two points $\mathrm{A}$ and $\mathrm{B}$ in the space of variables $x, y_{1}, . ., y_{n}$ through which an extremal must pass gives precisely $2 \mathrm{n}$ equations for determining these constants. Hence in the general case there appears a discrete set of extremals joining these points .Let $I_{A B}$ be the value of the functional on each of these extremal, $A$ being regarded as the initial and $\mathrm{B}$ as the terminal point. Let A be fixed while $B\left(x, y_{1}, y_{2}, \ldots, y_{n}\right)$ is regarded as a movable point. Then $I_{A B}$ is a function of $\left(x, y_{1}, y_{2}, \ldots, y_{n}\right)$ and we write

$$
I_{A B}=S\left(x, y_{1}, y_{2}, \ldots, y_{n}\right)
$$

If $B$ changes its position (6) gives

$$
d S=-H d x+\sum_{i=1}^{n} p_{i} d y_{i}
$$

Which in turn leads to $\frac{\partial S}{\partial x}=-H \quad, \frac{\partial S}{\partial y_{i}}=p_{i} \quad i=1.2, \ldots, n$

It then follows that $S$ satisfies the following partial differential equation of first order

$$
\frac{\partial S}{\partial x}+H\left(x, y_{1}, y_{2}, . ., y_{n}, \frac{\partial S}{\partial y_{1}}, . ., \frac{\partial S}{\partial y_{n}}\right)=0
$$

Which is known as Hamiltonian -Jacobi equation.

\subsection{3-Schrödinger Equation and Variatinal principle}

Now we derive the fundamental equation of quantum mechanics (Schrödinger equation) from a variational principle.

First we define an operator known as the Hamiltonian operator as follows:

$$
H \equiv-k \nabla^{2}+V(x, y, z)
$$

Here $k=h^{2} /\left(8 \pi^{2} m\right)$, where $h$ and $m$ stand for the Plank's constant the mass of the principle whose motion is considered in a field of potential energy $V$. We now seek a ware function $\Psi$

Possibly complex extremize the functional

$$
\iiint \Psi^{*}(H \Psi) d x d y d z
$$

Subject to the constraint

$$
\iiint \Psi^{*} \Psi d x d y d z=1
$$

Where $\Psi^{*}$ is the complex conjugate of $\Psi$.The integration is over a fixed domain of $x, y$ and $z$, We further assume that the admissible function $\Psi$ and $\Psi^{*}$ either vanish at corresponding points on opposite boundaries. As a consequence

\section{$\iiint \Psi^{*} \nabla^{2} \Psi d x d y d z=-\iiint \nabla \Psi^{*} \cdot \nabla \Psi d x d y d z$}

Introducing Lagrange multiplier $\lambda$, we then find the extremum of the functional

$\iiint K d x d y d z=\iiint\left[k\left(\Psi_{x}^{*} \Psi_{x}+\Psi_{y}^{*}+\Psi_{z}^{*} \Psi_{z}\right)+V \Psi^{*} \Psi-\lambda \Psi^{*} \Psi\right] d x d y d z$

The Euler equation are

$\frac{\partial K}{\partial \Psi}-\frac{\partial}{\partial x}\left(\frac{\partial K}{\partial \Psi_{x}}\right)-\frac{\partial}{\partial y}\left(\frac{\partial K}{\partial \Psi_{y}}\right)-\frac{\partial}{\partial z}\left(\frac{\partial K}{\partial \Psi_{z}}\right)=0$,

$\frac{\partial K}{\partial \Psi^{*}}-\frac{\partial}{\partial x}\left(\frac{\partial K}{\partial \Psi_{x}^{*}}\right)-\frac{\partial}{\partial y}\left(\frac{\partial K}{\partial \Psi_{y}^{*}}\right)-\frac{\partial}{\partial z}\left(\frac{\partial K}{\partial \Psi_{z}^{*}}\right)=0$,

Which reduce to 


$$
-k \nabla^{2} \Psi+V \Psi=\lambda \Psi
$$

This is written as $H \Psi=\lambda \Psi$

If we multiply this by $\Psi^{*}$ and integrate over the domain of $x, y, z$, the left side becomes the stationary integral (11) which depend by $E$.Hence by (12) we have $\lambda=E$, so (13) reduces to Schrödinger equation. It is worth pointing out here that there is an interesting and important connection between Hamiltonian-jacobi equation for classical system and the Schrödinger equation for a quantum mechanical system .In fact ,if we put the wave function $\Psi=e^{(i / h) S}$, where $S$ is the action function of the classical system (8),then the Schrödinger equation reduces to the Hamiltonian -Jacobi equation(9) provided $S$ is much larger than Plank's constant $h$.Thus in the limit of large values of action and energy, the surfaces of constant phase for the wave function $\Psi$ reduce to surfaces of constant action $S$ for the corresponding classical system. In this case, wave mechanics reduces to classical mechanics just as wave optics reduces to geometrical optics in the limit of very small wavelength. It may be noted that the KleinGordon equation

$\nabla^{2} \Psi-\frac{1}{c^{2}} \frac{\partial^{2} \Psi}{\partial t^{2}}-\left(\frac{m c}{h}\right)^{2} \Psi=0$,

( $\mathrm{c}=$ velocity of light) representing a possible wave equation for a relativistic particle (though it is not correct for an electron or proton)can be constructed in

$$
L=-\frac{h^{2}}{2 m}\left[\nabla \Psi^{*} \cdot \nabla \Psi-\frac{1}{c^{2}}\left(\frac{\partial \Psi}{\partial t}\right)^{2}+\left[\frac{m c}{h}\right]^{2} \Psi^{*} \Psi\right]
$$

\section{Conclusion}

The main result of this research we deduced Schrödinger equation by using variational principle and additional result we deduced Schrödinger equation is reduces to the Hamiltonian -Jacobi equation.

\section{References}

[1] Abraham Albert Ungar - Analytic Hyperbolic Geometry and Albert Einstein's Special Theory Relativity-World Scientific publishing Co-Pte.Ltd, (2008).

[2] Al Fred Grany, Modern Differential Geometry of Curves and Surfaces with Mathematica, CRC Press (1998).

[3] Aubin Thierry, Differential Geometry-American Mathematical Society (2001).

[4] Aurel Bejancu \&Hani Reda Faran-Foliations and Geometric Structures, Springer Adordrecht, the Netherlands (2006).

[5] Bluman G.W \& Kumei. S, Symmetry and Differential Equations New York: Springer-Verlag (1998).

[6] David Bleaker-Gauge Theory and Variational Principle, Addison- Wesley Publishing Company, (1981).

[7] Differential Geometry and the calculus of Variations, Report Hermann-New York and London, (1968).

[8] Edmund Bertschinger-Introduction to Tenser Calculus for General Relativity, (2002).

[9] M. Lee. John-Introduction to Smooth Manifolds-Springer Verlag, (2002).

[10] Elsgolts,L., Differential Equations and Calculus of variations, Mir Publishers,Moscow,1973.

[11] Lyusternik,L,A., The shortest Lines:Varitional Problems, Mir Publishers,Moscow,1976.

[12] Courant, R. and Hilbert,D. ,Methods of Mathematical Physics, Vols. 1 and 2,Wiley - Interscince, New York, 1953.

[13] Hardy,G.,Littlewood,J.E.and Polya,G.,Inqualities,(Paperback edition),Cambrige University Press,London,1988.

[14] Tonti,E.,Int. J. Engineering Sci.,22,P.1343,1984.

[15] Vladimirov,V.S.,A Collection of problems of the Equations of Mathematical Physics, Mir Publishers,Moscow,1986.

[16] Komkov,V.,Variational Principles of Continuum Mechanics with Engineering Applications,Vol.1,D.Reidel Publishing Co.,Dordecht,Holland, 1985.

[17] Nirenberg,L.,Topic in Calculus of Variations (edited by M.Giaquinta),P.100,Springer - Verlag,Berlin 1989.

\section{Biography}

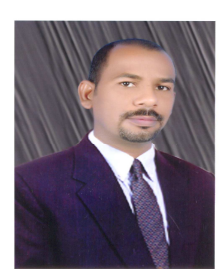

Dr. Sami Hajazi Mustafa: received his $\mathrm{PhD}$ degree in Differential Geometry from Alneelain University, Sudan in 2007. He was a head of Department of Mathematics in Academy of Engineering Sciences in Sudan. Now he is Assistant Professor of Mathematics, University college of Alqunfudha, Umm Al Qura University, Saudi Arabia. 